\title{
Track Reconstruction Using the Hough Transform at BESIII *
}

\section{Y. Zhang}

Institute of High Energy Physics, Chinese Academy of Sciences

E-mail: zhangyao@ihep.ac.cn

\section{J. Zhang}

Institute of High Energy Physics, University of Chinese Academy of Sciences

E-mail: zhangjin@ihep.ac.cn

\section{Y. Yuan}

Institute of High Energy Physics, Chinese Academy of Sciences

E-mail: yuany@ihep.ac.cn

\section{H.M. Liu}

Institute of High Energy Physics, Chinese Academy of Sciences

E-mail: liuhmeihep.ac.cn

In order to overcome the difficulties caused by the curling charged tracks in the BESIII drift chamber, we introduce a Hough-transform-based tracking algorithm, which is used as a supplementary method to find low transverse momentum tracks. The Hough transform is a mathematical method that can map hits in a detector to parameter space to find tracks globally. This tracking algorithm is implemented in the BESIII offline software system, and its performance is checked using both Monte Carlo and data. The results show that this tracking method improves reconstruction efficiency at the low transverse momentum region.

ICHEP 2018, 39th International Conference on High Energy Physics

4-11 July 2018

Seoul, Korea

*National Key Basic Research Program of China under Contract No. 2015CB856706; National Natural Science Foundation (NSFC) of China under Contracts Nos. 11475208, 11775245, 11335009, U1632106, 11575222 ; Chinese Academy of Sciences (CAS) President's International Fellowship Initiative under Contract No. 2018PM0004 


\section{Introduction}

The Multilayer Drift Chamber (MDC) is the main tracking detector for the Beijing Spectrometer III ( BESIII [1], Fig. 1(a) ). The tracking efficiency for MDC is high [2] for high transverse momentum $\left(\mathrm{p}_{\mathrm{T}}\right)$ charged particles but lower when $\left(\mathrm{p}_{\mathrm{T}}\right)<120 \mathrm{MeV} / \mathrm{c}$ as shown in Fig. 1(b), where some curling tracks may not be reconstructed, as shown in Fig. 1(c).

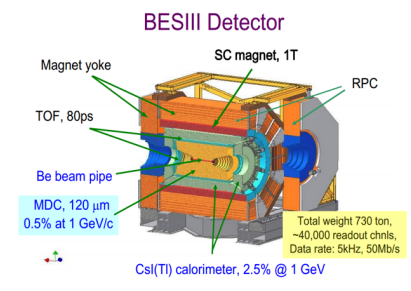

(a)

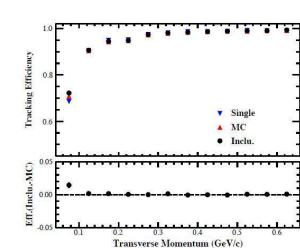

(b)

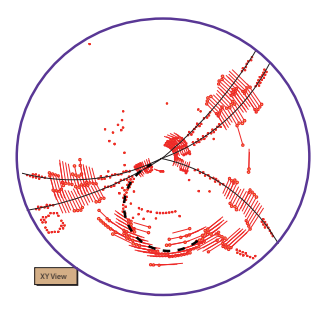

(c)

Figure 1: (a) BESIII detector. (b) $\pi^{+}$tracking efficiency at BESIII. (c) An event recorded at BESIII with a track not reconstructed in the dashed curve.

There are several tracking packages used in offline track reconstruction at BESIII, however all of them are local track finders which firstly search track segments in super-layers then link them to tracks. Hough transform is a mathematical method that can transform pattern in the geometric space to parameter space, in which we can find the pattern more easily. In MDC, a conformal mapping method is firstly used to transform circular track to the linear track on the conformal plane, then a extended Hough transform with drift circles is implemented. In this paper, we demonstrate a global track finder based on the Hough transform [3] and use it as a supplement at low $\mathrm{p}_{\mathrm{T}}$ region.

\section{Track Reconstruction}

With conformal mapping, the task of finding a circular track on the $x-y$ detector plane becomes one of finding a straight line on the conformal plane. Considering the drift distance, we use the extended Hough transform [5] which maps the lines tangent to the drift circle to curves in the Hough space, as shown in Fig. 4. The transformation is described by the equations 2.1,

$$
\begin{gathered}
\rho=X \cos \alpha+Y \sin \alpha+r,(\text { for upper side of circle }) \\
\rho=X \cos \alpha+Y \sin \alpha-r .(\text { for lower side of circle })
\end{gathered}
$$

A 2-D histogram is filled in the Hough space by axial-wire hits, followed by a peak finding method to investigate the histogram, and the peaks found are associated to 2-D candidate tracks. For 3-D track finding, we use the stereo-wire hits to march the tracks from 2-D track, with the left-right ambiguity taken into account. After 2-D and 3-D track finding, the tracks are fitted in a least square method and then merged with the exited tracking algorithm. Material effects as well as the field non-uniformity are corrected by the Runge-Kutta and Kalman filter algorithms.

\section{Tracking Performance}

We select the control sample from $J / \psi \rightarrow p \bar{p} \pi^{+} \pi^{-}$by requiring three charged tracks be identified, and then check if the fourth candidate track is reconstructed [2]. The tracking efficiencies of 


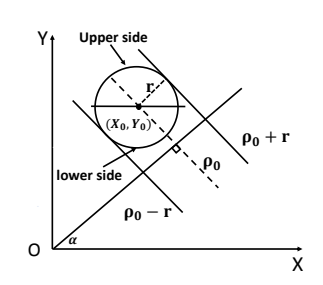

(a) X-Y conformal plane

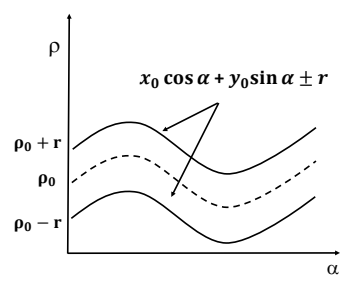

(b) parameter space

Figure 2: (a) A drift circle and two examples of tangent lines in the $\alpha$ direction on conformal plane. (b) Representation of the drift circle in the Hough space.

$\pi^{+}$are compared with and without the Hough transform for experimental data and MC simulation as shown in Fig. 3(a) and Fig. 3(b). The average increase with the Hough algorithm is about 7\%.

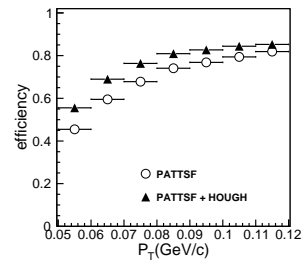

(a)

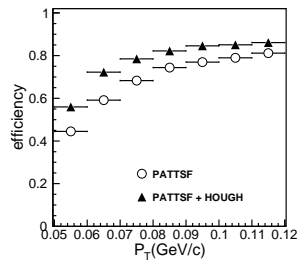

(b)

Figure 3: $\pi^{+}$efficiency of (a) data and (b) exclusive MC simulation in $J / \psi \rightarrow p \bar{p} \pi^{+} \pi^{-}$as a function of $\mathrm{p}_{\mathrm{T}}$ with and without the HOUGH algorithm.

The fake track rate is studied by single-particle MC event sample. An event with fake tracks is defined by if more than one track are reconstructed. The rate of fake track events increases from $0.3 \%$ to $0.6 \%$ when the Hough algorithm is used.

\section{Summary and Outlook}

In order to overcome the difficulties caused by the curling tracks at BESIII, we have developed an algorithm based on the Hough transform and used it as a supplementary method for low $\mathrm{p}_{\mathrm{T}}$ tracking. This method improves reconstruction efficiency at about $7 \%$ when $\mathrm{p}_{\mathrm{T}}$ is less than 120 $\mathrm{MeV} / \mathrm{c}$. We are trying to extend the algorithm to high $\mathrm{p}_{\mathrm{T}}$ region toward an unified tracking package.

\section{References}

[1] Design and construction of the BESIII detector, BESIII colaboration, 2010:347

[2] W. L. Yuan, et al., Chin. Phys. C 40 (2), 026201, 2016.

[3] P.V.C Hough, US Patent 3,069,654 (1962)

[4] R.D. Duda, P.E. Hart, Communications of the ACM 15(1), 11 (1972)

[5] T. Alexopoulos, et al., Nucl. Instr. and Meth. A 592 (3), 456, 2008. 